\title{
Bedside Communication and Management of Vital Parameters and Alarms In Care-Intensive Environments: Simulation Model Development for the Clinical Effectiveness Analysis of an Innovative Technology
}

\author{
By I. De Rosa', A. Pepino', G. Giaconia”, M. Guarino ${ }^{3}$ \\ ${ }^{1}$ Biomedical Engineer - Università degli Studi di Napoli Federico II \\ ${ }^{2}$ Professor of bioengineering - Università degli Studi di Napoli Federico II \\ ${ }^{3}$ Director of Economato and Clinical Engineering - Azienda Ospedaliera dei Colli di Napoli \\ ${ }^{4}$ Director of Emergency Department - Azienda Ospedaliera dei Colli di Napoli.
}

\section{ABSTRACT}

Background and Objective: The deliberation n.7301 of 31/12/2001 provides for the inclusion of a call system with acoustic and luminous signaling within the minimum equipment of the recovery ward. However, traditional call systems are inefficient since they are based on the following incorrect assumptions: patients and staff are unmoving, information sources are static, and assistance is unidirectional. Taking care of a patient involves different personnel who should be dynamic and should be able to exchange information. Furthermore, the high number of clinical calls and alarms might be an issue, as they are essential to fulfill patients' needs, but could cause stress and additional workload for medical staff. Indeed, they sometimes ignore some calls or waste time on non-urgent requests. Also, the identification of an alarm and prompt intervention seems to be more difficult during travel. An ideal alarm system should have $100 \%$ sensitivity and specificity. However, the alarms are designed to be extremely sensitive, at the expense of specificity. The alarm fatigue, that is the work overload due to an excessive alarms number exposition, is a critical problem in terms of safety in the current clinical practice because it involves desensitization and alarm loss, and occasionally a patient's death.

Material and Methods: Appropriate approaches to notifications should be evaluated, including the effectiveness of mobile wireless technologies that are key to linking patients, staff, data, services, and medical devices which simplifies communications and workflows. Several issues related to the communication among staff members, between patient and caregiver, and regarding the alarms and vital parameters distribution in care-intensive environments have been analyzed. The focus was on the clinical effectiveness analysis of innovative technology to support the activities in the Emergency Department of the Azienda Ospedaliera dei Colli. Afterward, we created a simulation model with Simul8, so that a digital twin reproduces direct and indirect activities in two cases: with and without (What If and As Is model) the aid of the technology.

Results and Conclusions: The model provides a set of Key Performance Indicators (number of performing activities, average alarm resolution time, wait time) on which the compensatory aggregation method is applied to obtain a single final score in both cases. This score is 52.5 in the As Is Model and 80 in the What If model. So, clinical effectiveness has been demonstrated.

Keywords - Alarm fatigue, safety, communication, clinical effectiveness, simulation model, workflow, vitalsigns.

Copyright (C) 2021. This is an open-access article distributed under the terms of the Creative Commons Attribution License (CC BY): Creative Commons - Attribution 4.0 International - CC BY 4.0. The use, distribution or reproduction in other forums is permitted, provided the original author(s) and the copyright owner(s) are credited and that the original publication in this journal is cited, in accordance with accepted academic practice. No use, distribution or reproduction is permitted which does not comply with these terms. 


\section{INTRODUCTION}

Different people are involved in the patient care process. All of them have to share and discuss information about patient management. These people are not stationary but move around the hospital while engaging in multiple activities at the same time. This can include the manual recording of clinical data and filing medical records which increases the possibility of error and can impact the assistance response times. Furthermore, hearing and correctly identifying an alarm signal and promptly intervening can be more difficult due to the movement of caregivers. As a result, both the interest and the use of information and communication technologies to support health services has increased.

Information and communication technologies offer powerful tools to restructure health service processes. Nowadays, there is a growing range of communication channels, media, and devices, which makes it possible to provide these services. A growing literature on the value of communication in the healthcare sector has already been developed.

Although there has been advanced research in highly specific areas (i.e., telemedicine), the clinical adoption of simpler services, such as voice mail or email, are still not common in many health services. This situation would change if we realized that the biggest information repository in healthcare is the heads of the people who work in it, and the biggest information network is the complex network of conversations that connects the actions of these individuals. ${ }^{1}$

Even small clinical teams can generate large and complex communication spaces. The clinical communication space is also characterized by numerous interruptions, poor communication systems, and inadequate practices.

The participants are often separated by time and space. We have synchronous communication in case the attendees exchange messages simultaneously, asynchronous if not (Table 1).

Therefore, care devices and hospital information systems should be integrated to encourage the exchange of information among caregivers and to provide structured data for improving the timely and effective coordination of care.
TABLE 1. Values Measured in a Patient Session

\begin{tabular}{|c|c|c|c|}
\hline synchronous & Sound & Images & Data \\
\hline asynchronous & Voice mail & $\begin{array}{c}\text { Video } \\
\text { conferencing } \\
\text { image archive }\end{array}$ & $\begin{array}{c}\text { Electronic } \\
\text { cards, } \\
\text { documents }\end{array}$ \\
\hline
\end{tabular}

The goal is to provide an easy way to acquire and insert clinical data into the hospital registration system, through the use of mobile, lightweight, portable devices. Linking patients, staff, data, services and medical devices simplifies communications and workflows.

Appropriate approaches to notifications should be evaluated, including the effectiveness of mobile wireless technologies to reduce alarm fatigue. The analysis is focused on the current structure and organization of the Emergency Department of Hospital CTO of Napoli (Azienda Ospedaliera dei Colli of Napoli).

This work aims to propose and test a new organizational, technological, and managerial network which is capable of optimizing the hospital's response to the individual's need for health and guarantee caregivers the ability to carry out their clinical activities within the system

The study involves the analysis of the clinical effectiveness (one of the nine domains defined by the EUnetHTA Core Model) of a technology that supports the department activities. For this analysis, a digital twin of the healthcare process was developed using Simul8, to which a set of indicators was calculated. Finally, the compensatory aggregation method was applied to the selected indicators, to obtain a single value that allowed the evaluation of the clinical effectiveness of this technology.

\section{STATE OF ART}

In this work, studies and solutions in literature that face these problems have been analyzed. One of the most representative is Hendrich's study. ${ }^{2}$ After equipping each nurse with a Personal Digital Assistant (PDA) for recording activities and a bracelet capable of measuring skin temperature and displacements to assess energy expenditure and distances traveled, this study showed that a 
nurse spends $37 \%$ of her time in the patient's room and $43 \%$ in the nursing station (Figure 1).

\section{Nurse activities: Location}

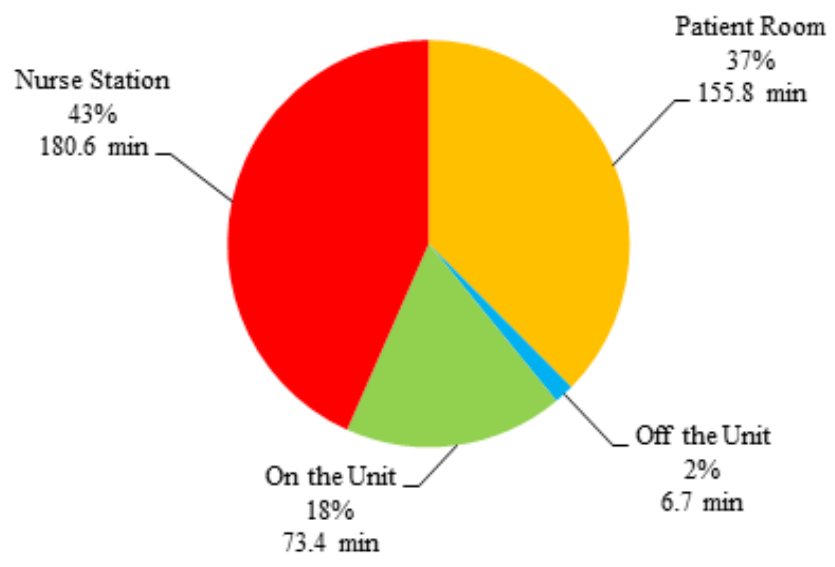

FIGURE 1. Nurse activities: Location.

The main nurse activities are the documentation (i.e., the compilation of medical records, acceptance, and discharge documents) and the coordination of the treatment process (i.e., the communication with other team members to establish the best approach for the patient). A total of $19 \%$ of their time (less than $1 / 5$ of the time) was dedicated to direct patient care activities and only 7\% was dedicated to the monitoring of vital signs (Figure 2).

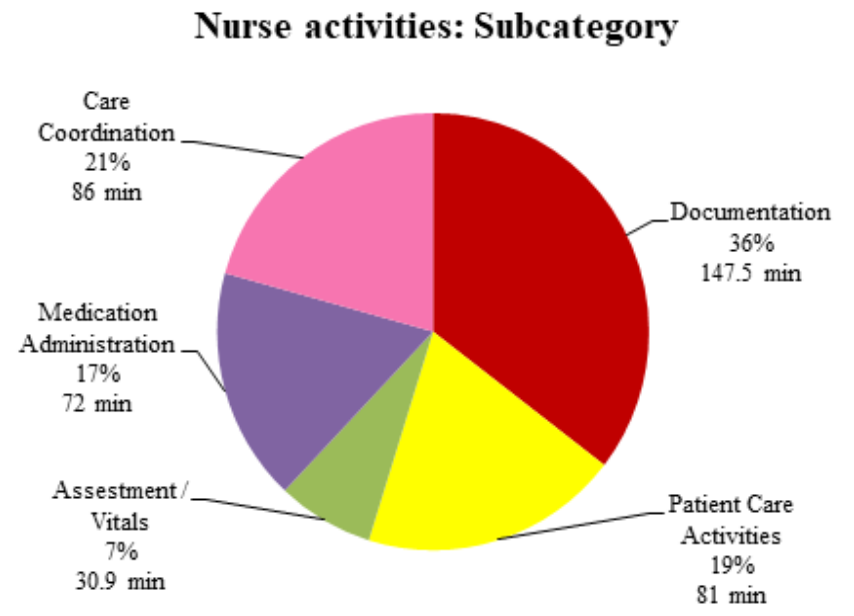

FIGURE 2. Nurse activities: Subcategory.

The Manhattan Medical Research Adoption Study of June 2012 found that the use of mobile devices in healthcare is pervasive (Figure 3). The majority of the interviewed clinicians (87\%) confirmed the adoption of smartphones and tablets in the workplace to improve resources and information at the point of care. ${ }^{3}$

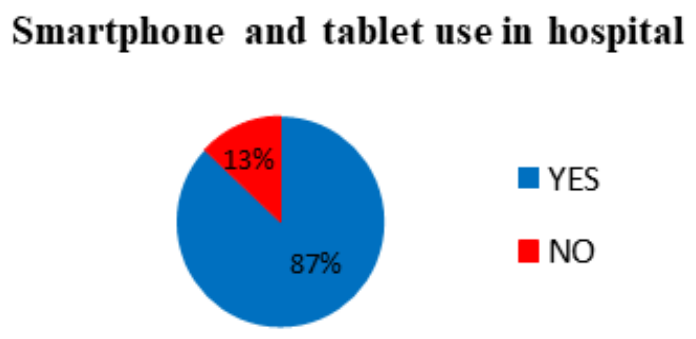

FIGURE 3. Smartphone and tablet use in hospital.

Company policies for the use of mobile devices can be BYOD (Bring Your Own Device) or COPE (Corporate Owned, Personally Enabled). In the first case, the company's initial investment is less, but there are lots of hidden costs and risks, such as distractions, which can lead to clinical risk situations, cybersecurity issues, and data loss problems.

In the hospital, it would be appropriate to provide caregivers with dedicated devices. The main features of these devices should be that they are high quality, lightweight to support mobility; robust and resistant to the action of aggressive detergents or disinfection solvents to reduce infections; impermeable, have a longer battery life, and good network coverage.

Another important problem is alarm fatigue. Sendelbach's research showed that from $72-99 \%$ of clinical alarms are false alarms. ${ }^{4}$ The high number of false alarms has led to the alarm fatigue problem. Alarm fatigue an overload of work that occurs when caregivers are exposed to an excessive number of alarms it may lead to desensitization and loss of the alarms.

The research should evaluate various approaches to alarm notification, including the effectiveness of wireless technology and to increase the specificity of the alarms without a significant loss of sensitivity. This research aims to figure out these problems by focusing on the analysis of the clinical effectiveness of innovative technology in support of the ward activities.

The technology is modular and includes patient receivers, bed modules, conversation modules (to allow patients to quickly communicate with caregivers and control the 
environment), modules with inputs to connect medical devices for monitoring remotely and for the alarm notification, door modules, peripheral modules, corridor lights and displays, personnel consoles, signalers, gateways, and passive bus concentrators (Figure 4 ). ${ }^{5}$

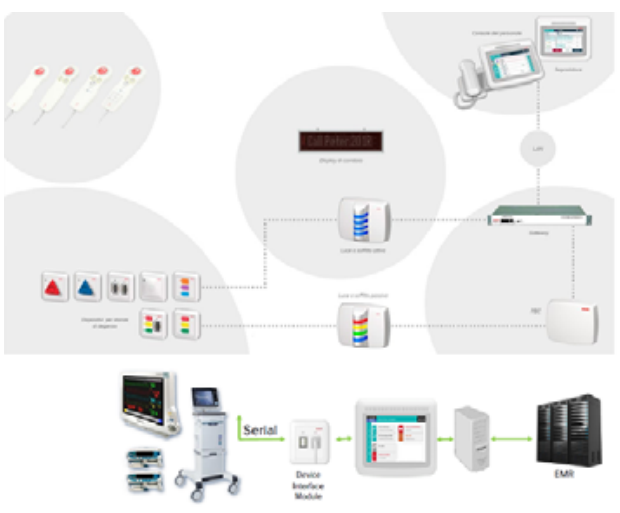

FIGURE 4. Ascom Telligence technology.

\section{METHODS}

Clinical effectiveness is one of the nine domains defined in the EUnetHTA Core Model; a multidisciplinary evaluation model born from the EUnetHTA project funded by the European Union since 2006. The nine domains are developed by a multidisciplinary and multi-professional team.

This work involves the analysis of the fourth domain: the evaluation of the clinical effectiveness of the technology. Effectiveness represents the benefit obtained by using technology in a real work context, whereas the efficacy represents the benefit in ideal conditions.

Simulation can be seen as a valid method for assessing effectiveness, especially in situations where there is a lack of data in the literature and there is no possibility of directly observing the use of technology. Simulating consists of reproducing as accurately as possible the functioning of a system to study its responses to the change of the external environment, even before putting the change into action, through the analysis of suitably chosen performance indicators, called Key Performance Indicators (KPI).

For this analysis, a digital twin of the healthcare process is developed using Simul8. A digital twin is a digital replica of physical systems, devices, processes, people, places. Simul8 is a simulation software product by SIMUL8 Corporation, used for the simulation of systems that involve the processing of discrete entities in discrete time. Through a model developed with Simul8, it is possible to test real scenarios in a virtual environment. Simul8 allows simulation of the process, defining activities, times, resources, work shifts, to obtain a model representing the entire workflow with reasonable reliability. A Simul8 simulation revolves around the processing of work items. They enter the system through the work entry points, pass through the work centers, can temporarily reside in the queues (storage areas), and terminate their path in the process through the work exit points. The work centers may need specific resources to process the represented activity. Simul8 outputs can be graphs, statistics, numeric values.

For this analysis, the analyzed KPIs are:

- number of performing direct activities compared to the total number of required direct activities;

- average alarm resolution time;

- waiting time.

It is possible to apply the compensatory aggregative method on them, to obtain a single decision support score.

$$
P=\sum_{i=1}^{N} w_{i} V_{i}=w_{1} V_{1}+w_{2} V_{2}+w_{3} V_{3}+w_{4} V_{4}+\cdots+w_{N} V_{N}
$$

$$
\begin{aligned}
& \mathrm{P}=\text { Priority score } \\
& \mathrm{w}_{-}(\mathrm{i})=\mathrm{i} \text {-th weight } \\
& \mathrm{V}_{-} \mathrm{i}=\mathrm{i} \text {-th indicator value }
\end{aligned}
$$

\section{CONTEST OF APPLICATION}

Mobile handheld devices show greater benefits in high care-intensive environments where time is critical and rapid response is crucial. The application context of the technology is the Emergency Department of Hospital CTO (Napoli). This consists of a First Aid located on the ground floor, equipped with 4 beds in the Observation Area, 2 beds in the red code room and 2 beds in the yellow code room (Figure 5).

The ward is located on the fourth floor and has 18 beds divided into 7 rooms ( 4 with 3 beds and 3 with 2 beds). There is also a nurse station (Figure 6). 


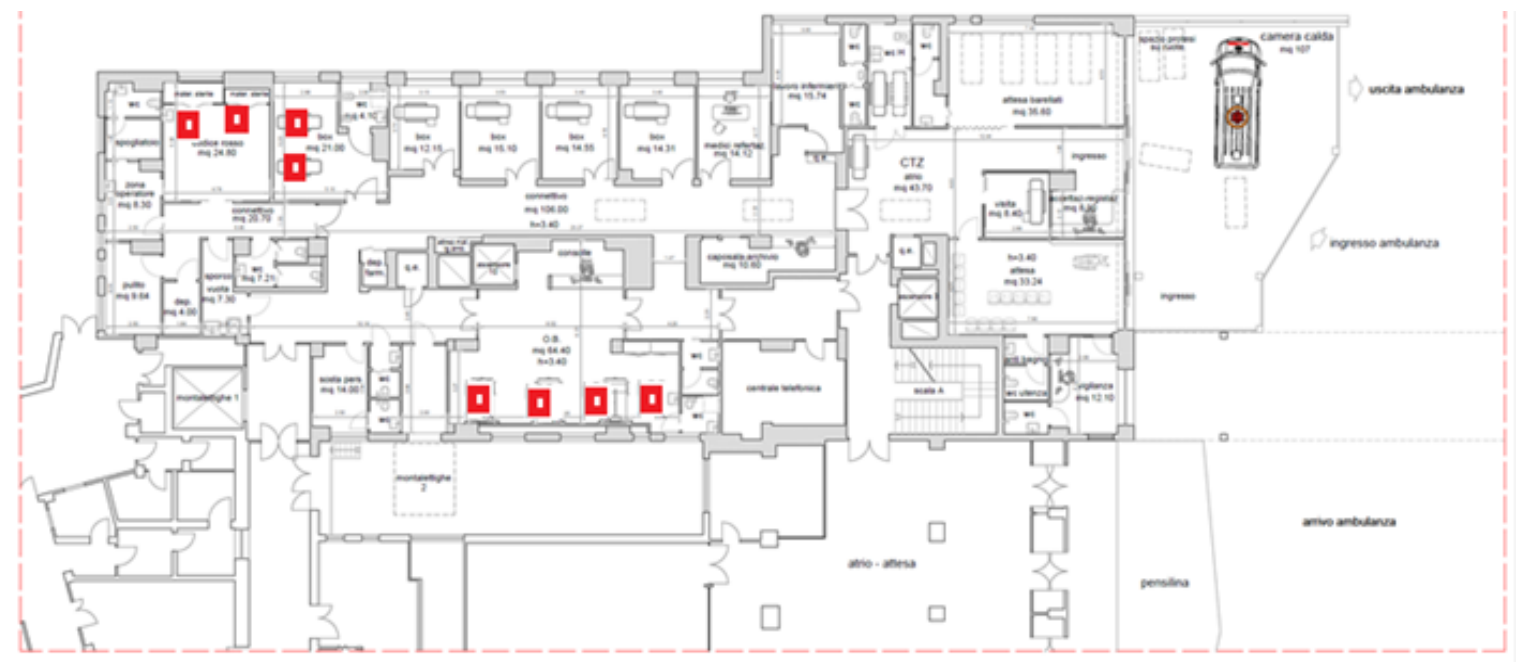

FIGURE 5. First Aid - ground floor.

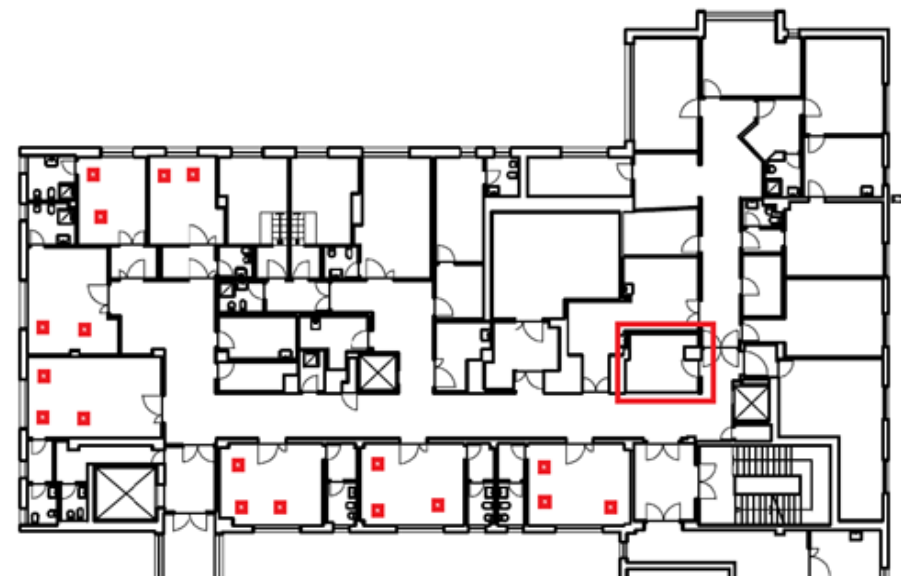

FIGURE 6. Emergency Department - fourth floor.

Considering the number of beds, it was determined that 26 devices should be installed (Table 2).

TABLE 2. Bed Locations

\begin{tabular}{|c|c|c|}
\hline & \multicolumn{2}{|c|}{ BEDS } \\
\hline First Aid & 4 & $\begin{array}{c}\text { 2 red code room } \\
\text { 2 yellow code room }\end{array}$ \\
\hline Observation Area & \multicolumn{2}{|c|}{4} \\
\hline Ward & \multicolumn{2}{|c|}{18} \\
\hline
\end{tabular}

Regarding the organization of the emergency-urgency team, in the worst case (the one with the highest number of caregivers) there were:
- 3 doctors, 8 nurses ( 2 of them are always in the triage area) and 1 social and health professional in the First Aid (ground floor), for a total of 12 units; and

- 2 doctors, 5 nurses and 1 social and health professional in the ward (fourth floor), for a total of 8 units.

The number of caregivers present at the same time was 20. It was determined that 20 smartphones should be given to caregivers (Table 3 ).

TABLE 3. Values Measured in a Patient Session

\begin{tabular}{|c|l|l|l|}
\hline & \multicolumn{1}{|c|}{ First aid } & Observation area & \multicolumn{1}{c|}{ Ward } \\
\hline \multirow{5}{*}{ Doctors } & $2(7: 00-15: 00)$ & $1(7: 00-15: 00)$ & $2(7: 00-15: 00)$ \\
& $2(15: 00-23: 00)$ & $1(15: 00-23: 00)$ & $1(15: 00-23: 00)$ \\
& $2(23: 00-7: 00)$ & $1(23: 00-7: 00)$ & $1(23: 00-7: 00)$ \\
\hline \multirow{3}{*}{ Nurses } & $7(7: 00-15: 00)$ & $1(7: 00-15: 00)$ & $5(7: 00-15: 00)$ \\
& $7(15: 00-23: 00)$ & $1(15: 00-23: 00)$ & $3(15: 00-23: 00)$ \\
& $6(23: 00-7: 00)$ & $1(23: 00-7: 00)$ & $3(23: 00-7: 00)$ \\
\hline \multirow{2}{*}{$\begin{array}{c}\text { Auxiliary } \\
\text { staff }\end{array}$} & $1(7: 00-15: 00)$ & $1(7: 00-15: 00)$ & \\
& $1(15: 00-23: 00)$ & $1(15: 00-23: 00)$ \\
$1(23: 00-7: 00)$ & $1(23: 00-7: 00)$ \\
\hline
\end{tabular}

\section{ELABORATION OF SIMULATION MODEL}

The development of the simulation model foresaw a first phase, in collaboration with the emergency medicine staff, in which all possible activities carried out in the ward were identified. During this analysis, several direct activities (completed at the bedside) and indirect activities were selected, in three different periods of the day 
(morning, afternoon, and night) which reflect the different work shifts and staff availability.

All staff members filled out a questionnaire. Their answers and the direct observation of the process allow the definition of the frequency and duration of their activities.

The direct activities identified were:

- $\quad$ therapy administration;

- vital parameters control;

- patient hygiene;

- $\quad$ withdrawal, catheterization, medications;

- tours;

- alarm management;

- $\quad$ bed calls; and

- health status updates.

The indirect activities identified were:

- $\quad$ emergency in the First Aid department;

- medical record filling;

- drug preparation and therapy;

- medication warehouse management;

- $\quad$ instrument management;

- $\quad$ briefing with colleagues;

- $\quad$ patient disposal activities;

- patient acceptance activity;

- conducting diagnostic tests;

- transfers to other facilities; and

- $\quad$ exam requests.

For direct activities, the work item corresponded to every care need at the bed. Every work item was processed in a work center and for each of them an operating time may be defined, depending on three different levels of patient complexity. Every activity was made by one or more people (nurses, doctors, and auxiliary staff) recruited on predefined shifts. These activities involve the movements of caregivers into the department and the operation time reflects the distance between the nurse station and the room from which the assistance need originated. Regarding indirect activities, the work items no longer represent patient needs but the repetitions of the individual activities and their duration was independent of the patient complexity level.

After having entered all the required data, the simulation model could run on different time frames and at different speeds through a dedicated cursor. It was also possible to obtain indications regarding the trend of the variables that characterize the functioning of the model through a series of graphs selected by the user. During execution, the icons and animations facilitate understanding of the workflow.

Before using the model, it was necessary to verify whether the model could represent a reasonable approximation of reality. An approach divided into two successive steps was adopted for its validation outlined below.

- Formal validation: evaluation of the code correctness.

- Structural validation: comparison between the behavior of the simulation model and the real system, to assess whether and how much the model can be considered a good approximation of reality. The structural validation consists of two successive moments: - open-box validation: the staff evaluate the model:

- black-box validation: the results are compared with the data obtained from the real system. ${ }^{6}$

According to these validations, it was possible to find that the simulation model implemented constituted a good approximation reality.

\section{THE AS IS MODEL}

The As Is model consists of the evaluation of the workflow characteristics and performance in the current configuration. The As Is model created is described in Figure 7.

To define the model, 5 steps needed to be implemented.

1. Identification of direct and indirect activities.

2. Identification of the frequency and duration of all activities through direct observation and questionnaire.

3. Identification of the resources that complete the activities, taking into account the work shifts.

4. Validation of the model.

5. Analysis of KPIs. 


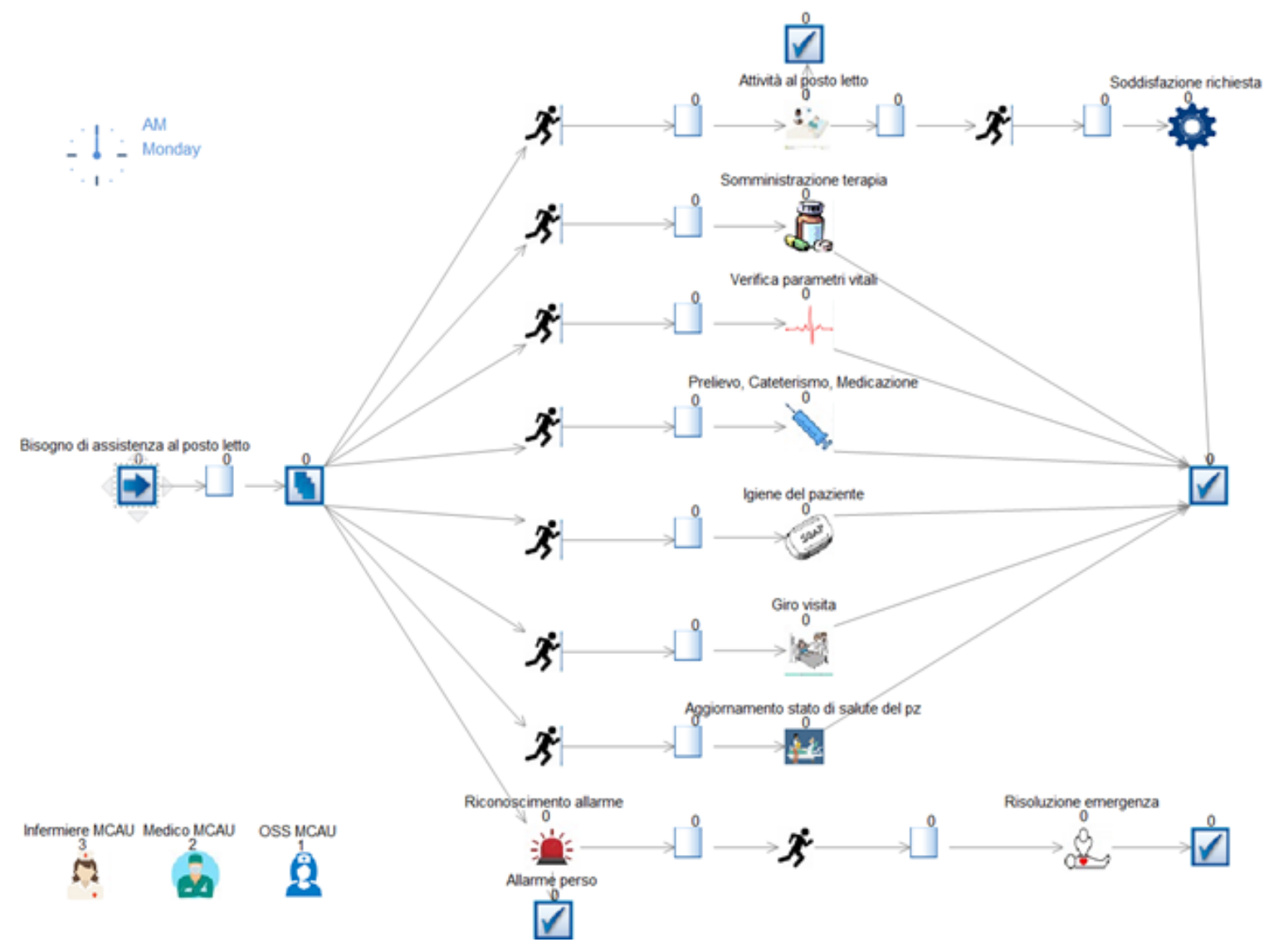

FIGURE 7. As Is model.

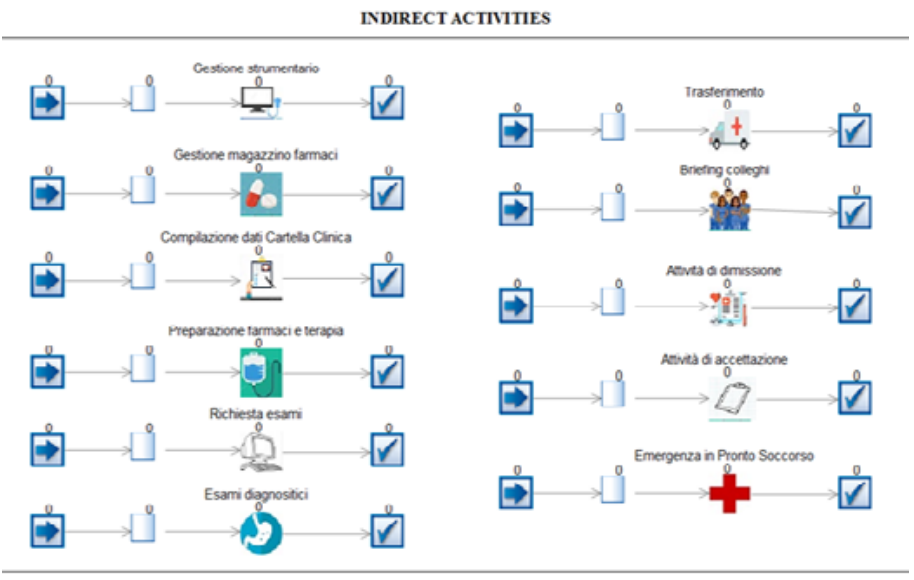

Each work item generated by the Start Point related to direct activities represented a care needs at the patient's bedside. Considering that there were 18 beds in the ward, it was estimated that these needs occurred every 5 minutes. A label was associated with each work item and set on a distribution that represents the frequency of each patient's bedside care needs (Figure 8).

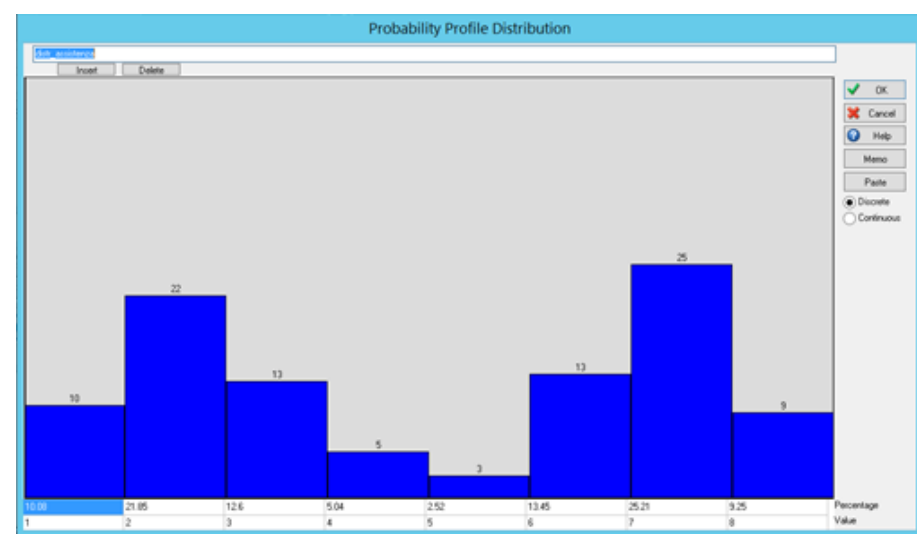

FIGURE 8. Distribution of patient bedside care needs.

Each care need may come from a different bed, so each generated work item was associated with an additional label, set on a different distribution which defined the distance from the nurse station and takes into account that rooms 1, 2, 3 and 4 have 3 beds and rooms 5, 6 and 7 have 2 beds (Figure 9). 


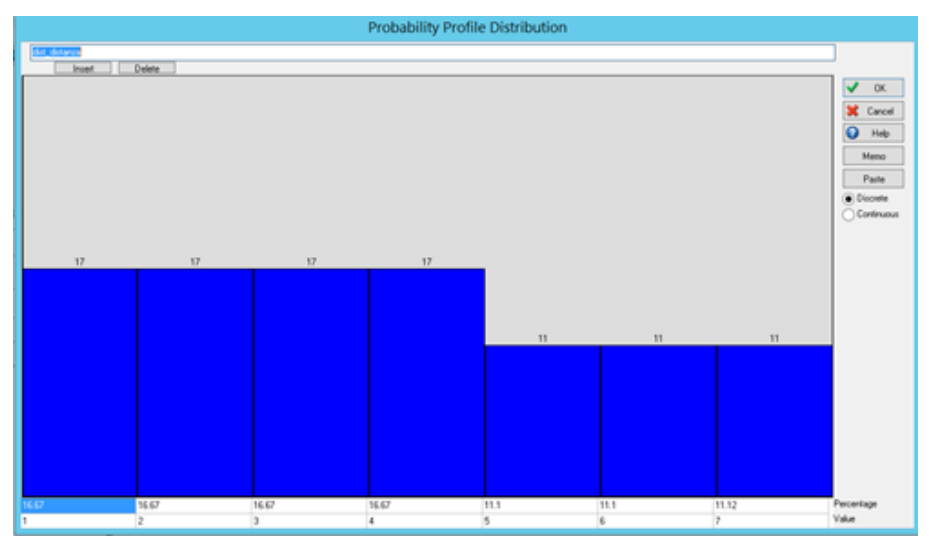

FIGURE 9. Distribution of nurse station-bed distances.

The movements were modeled through an activity whose operation time was defined by the time taken to reach the room the call comes from.

The 7 possible paths defining the movements from the nurse station to the patient's room have the following lengths (measured by AutoCAD): $15.64 \mathrm{~m} ; 23.82 \mathrm{~m} ; 30.62$ $\mathrm{m} ; 35.53 \mathrm{~m} ; 41.76 \mathrm{~m} ; 44.47 \mathrm{~m} ; 42.37 \mathrm{~m}$. Considering that the operator average speed is $5 \mathrm{~km} / \mathrm{h}$, the travel times are calculated. Operation Time is defined for the movement activities and depends on the distances (Figure 10).

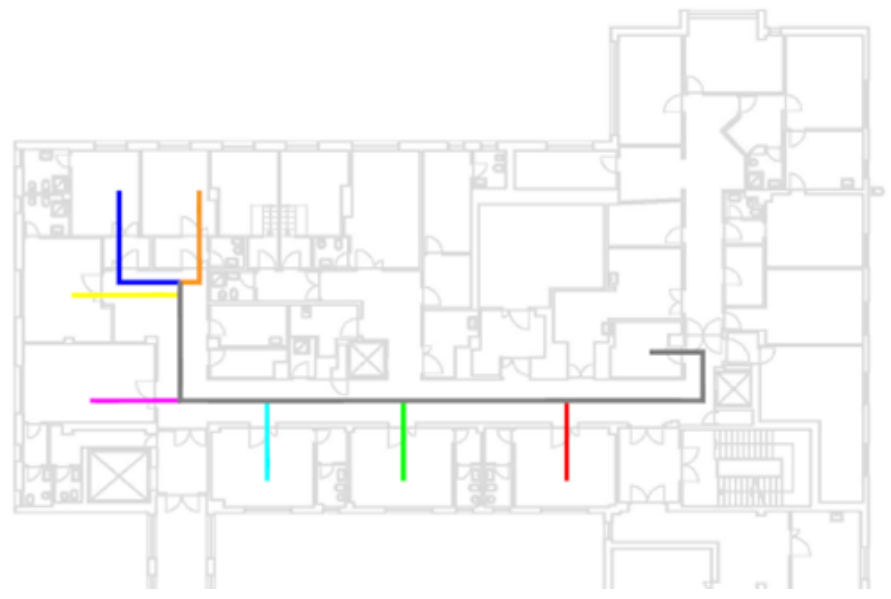

FIGURE 10. Routes on the Emergency department map.

Operation Times of the other activities, on the other hand, take into account the level of complexity of the patient (Figure 11).

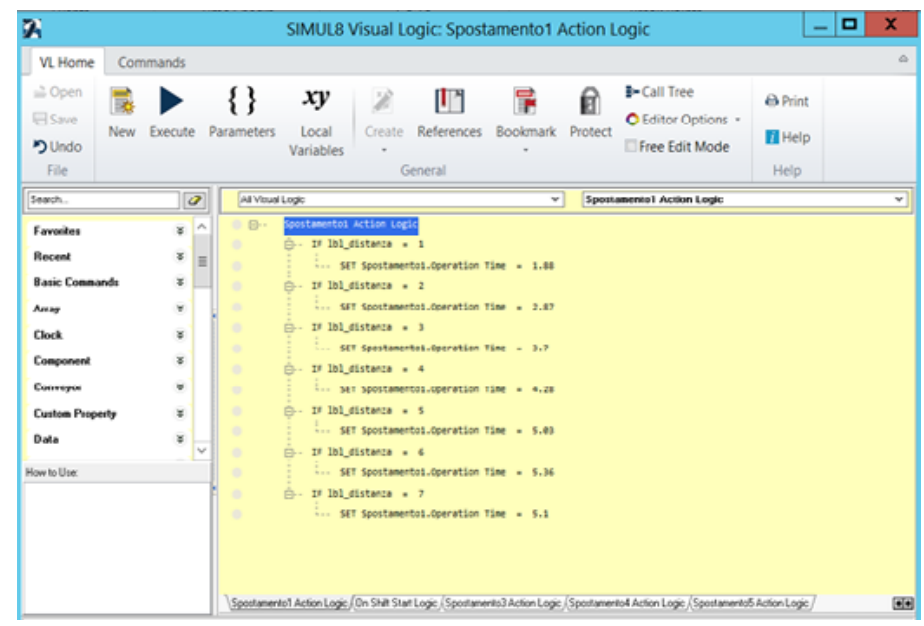

FIGURE 11. Visual Logic code for defining the movement activities Operation Times.

The model determined that only $50 \%$ of cases did the bedside activities end directly at the bedside: the caregiver was often forced to make multiple movements to satisfy the patient's needs. The model took into account the alarm acknowledgment times (due to the lack of a centralized monitoring system) and the possibility of losing the alarms (due to the alarm fatigue problem). Moreover, each activity was associated with one or more resources (Figure 12).

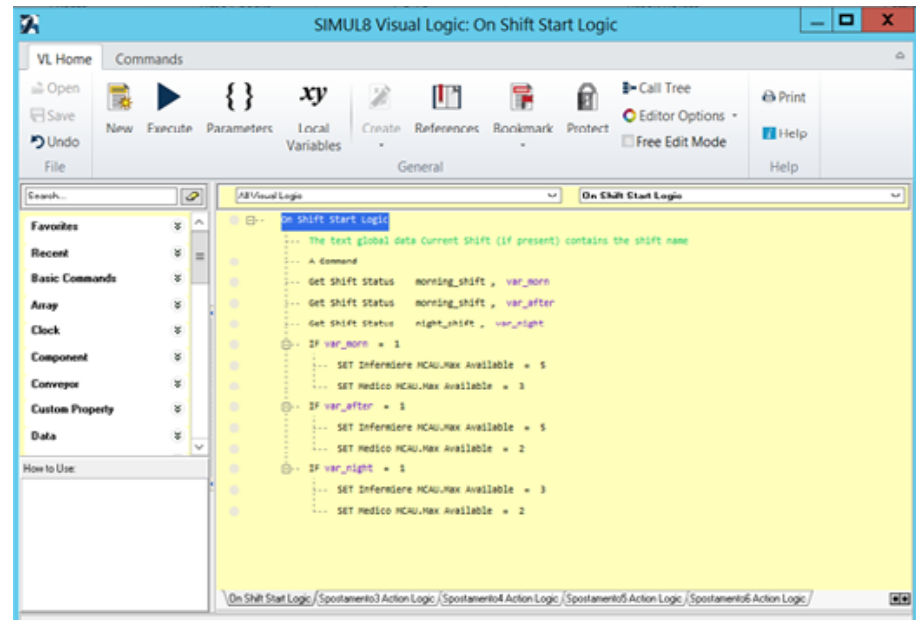

FIGURE 12. Visual Logic code for defining shifts.

Three shifts were identified.

1. Shift from 07.00 to 15.00 , in which 5 nurses and 3 doctors are available. 
2. Shift from 15.00 to 23.00 , in which 5 nurses and 2 doctors are available.

3. Shift from 23.00 to 07.00 , in which 3 nurses and 2 doctors are available.

One social and health professional was always available.

Regarding indirect activities, the work item no longer represented a patient's bedside needs but the single repetitions of the individual activities. Resources, frequency, and duration were also appropriately associated with the indirect activities and were independent of the patient complexity level.
The simulation time was 24 hours, every day for one month. According to the validation, the simulation model implemented constituted a reasonable approximation of the real system. It might, therefore, be suitably modified to study the response to the introduction of technology. Improvements to the workflow were introduced in the What If model to check the introduction of the technology's impact on KPIs.

\section{THE WHAT IF MODEL}

The What If model created is shown in Figure 13.

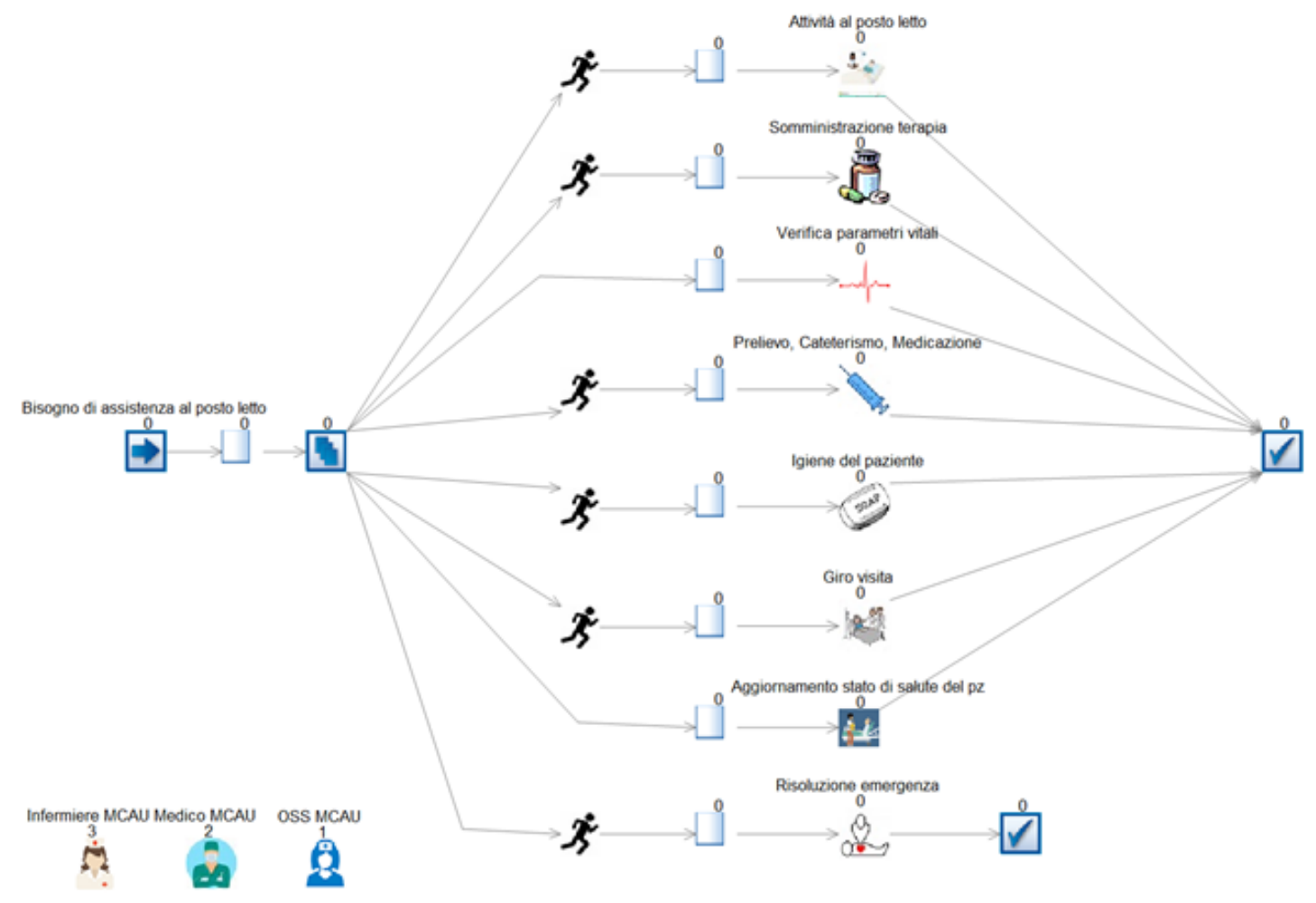

FIGURE 13. What If model.

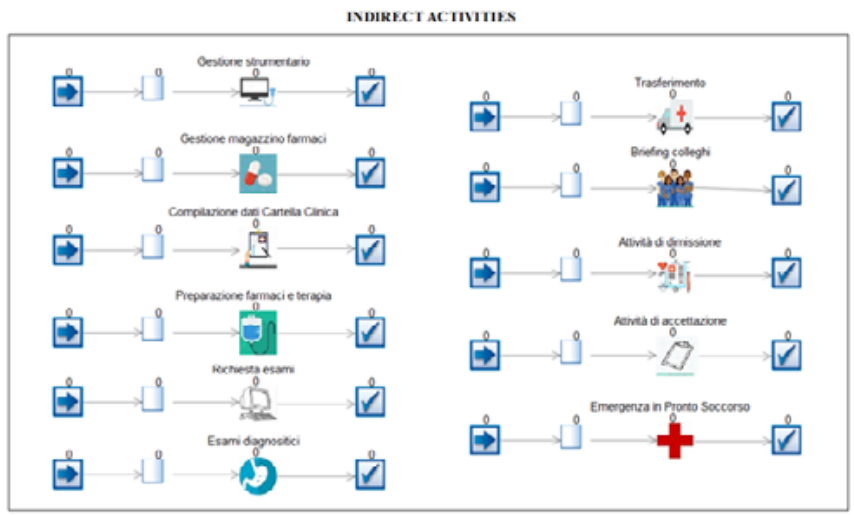

This model was created by properly modifying the As Is model, taking into account the activities on which the technology operates.

The main differences with the As Is model are:

1. The calls end directly to the bed, without making further movements. It may happen because the caregivers already know the patient's need even before going there.

2. The vital signs can be remotely checked.

3. The patient's health status can be updated via devices. 
4. There is no longer an alarm acknowledgment time since the technology reports the bed from which the alarm goes off. Furthermore, there are no more lost alarms. Indirect activities remain unchanged.

\section{RESULTS}

The KPIs are:

1. The number of completed direct activities compared to the required activities. The ratio between the two numbers ranges from 0 to 1 : it is 1 when the number of completed activities is equal to the required ones (ideal case), 0 if none of the required activities is completed (worst case).

$$
V_{1}=\frac{\text { number of completed activities }}{\text { number of required activities }}
$$

2. Average time to resolve an alarm.

$$
V_{2}=1-\frac{\text { Average alarm resolution time }}{\text { Maximum alarm resolution time }}
$$

3. Waiting time for direct activities.

$$
V_{3}=1-\frac{\text { Average waiting time }}{\text { Maximum waiting time }}
$$

The weights for the application of the compensatory aggregation method, chosen in collaboration with the staff, are: 50 for V1, 20 for V2, 30 for V3. The sum of the weights is 100 and the ideal values of V1, V2 and V3 is 1 .

At the end of the simulation, the data related to the identified KPIs are presented.

The As Is model showed the results below

1. A total of $70 \%$ of bedside care needs are fulfilled.

2. The average resolution time for an alarm was 28.57 minutes.

3. The waiting time to complete direct activities was 9.23 minutes.

KPIs for the As Is model are outlined in Figure 14 and the Compensatory Aggregation Method of the As Is Model is presented in Table 4.
TABLE 4. Bed Locations

\begin{tabular}{|c|c|c|c|}
\hline $\mathrm{Vi}$ & 0.7 & 0.065 & 0.54 \\
\hline wi & 50 & 20 & 30 \\
\hline \multicolumn{3}{|c|}{$0,7^{\star} 50+0,065^{\star} 20+0.54^{\star} 30=\mathbf{5 2 . 5}$} \\
\hline
\end{tabular}

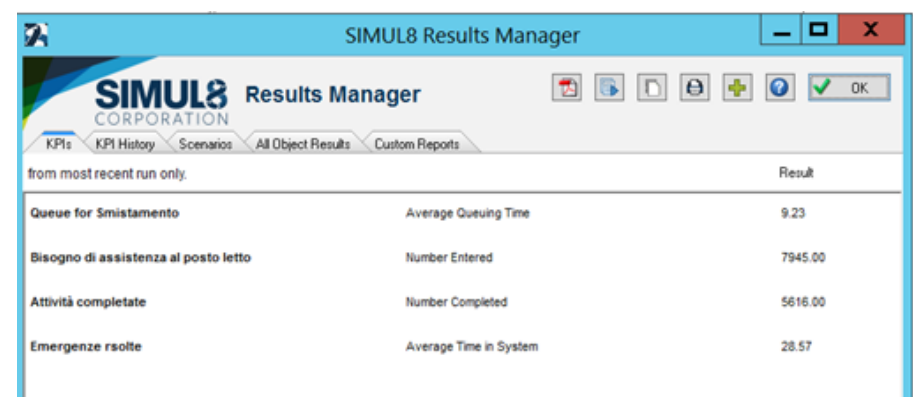

FIGURE 14. Compensatory Aggregation Method - As Is Model

The What If model shows the following results:

1. A total of $90 \%$ of bedside care needs are fulfilled.

2. The average resolution time for an alarm is $\mathbf{1 5 . 8 6}$ minutes.

3. The waiting time to complete direct activities is $\mathbf{5 . 1 6}$ minutes.

KPIs for the What If model are outlined in Figure 15 and the Compensatory Aggregation Method of the As Is Model is presented in Table 5.

TABLE 5. Compensatory Aggregation Method - What If Model

\begin{tabular}{|c|c|c|c|}
\hline Vi & 0.9 & 0.64 & 0.74 \\
\hline wi & 50 & 20 & 30 \\
\hline \multicolumn{3}{|c|}{$0,9 \star 50+0,64^{\star} 20+0,74^{\star} 30=\mathbf{8 0}$} \\
\hline
\end{tabular}

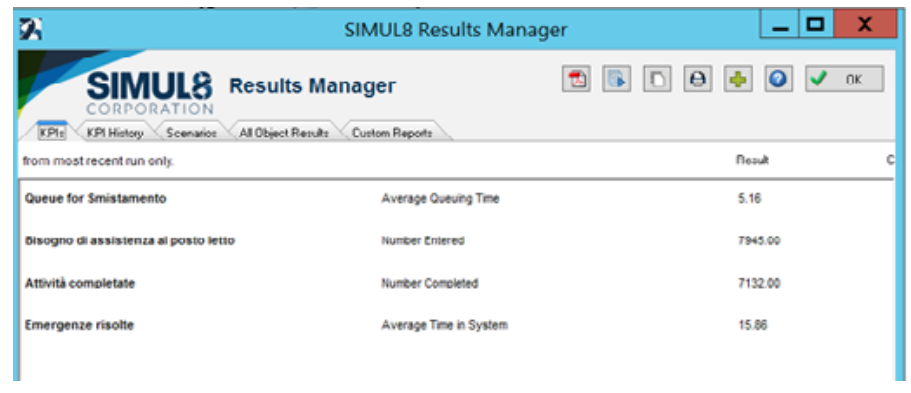

FIGURE 15. KPIs - What If model. 
Considering that in the ideal case the final score is 100 , from the compensatory aggregation method it results that this is 52.5 in the current model, 80 in the model that simulates the introduction of technology. The clinical effectiveness of the innovative technology for the communication and distribution of alarms and vital signs is therefore demonstrated.

\section{DISCUSSION}

The analysis of the clinical effectiveness of the technology studied in this work was based on the compensatory aggregation method applied on the KPIs obtained by the simulation models created with Simul8 (see Table 4 and Table 5).

The above-mentioned approach allows for:

- estimation of the organizational changes, which are generally complex to analyses in other ways;

- assessment of the operating conditions of the department;

- determination if, and how much, the resources operate in compliant conditions;

- determination of which resources intervene to improve the workflow; and

- determination of which activities should be modified.

This methodology was also very educative for the caregivers who had the opportunity to systematically analyzes their work organization both during the analysis phase and the discussion of the simulation results.

Having more precise measurement results of the real system behavior would be desirable: unfortunately, it is very complicated to obtain in an operating environment such as a hospital ward, even more in a high-intensity care environment like an emergency department. To obtain precise estimates, it would be necessary to measure the completed activities at the bedside in daily life with precise tools and for a longer time.

The main limitation of this work, like most of the works based on simulation models, is the difficulty to compare the results of the simulations with the results of the real world, despite the effort of the formal and structural validations. The analysis began from a real-world measurement of the process: it was empirical and based on subjective assessments of the caregivers and on the observation of ward activities. The reliability of the model and results depended on the reliability of the indications given by the caregivers and also the observations made internally in the ward. However, the model and methodology used can be considered a sufficient basis for further customizations in many case studies.

\section{CONCLUSIONS}

The clinical effectiveness of the technology supporting the ward activities was demonstrated with the simulation method, in situations in which validated scientific literature was not yet developed.

The As Is model has a good adherence to reality - both formal and structural validation were used. According to the caregivers, the As Is model represented a good approximation of reality, but the comparison should be made on indicators that can be accurately measured. It is not always possible, especially in emergency medicine departments. The What If model could be improved with the analysis of some data from realities where the technology is already in use.

The simulation model offers the possibility to find out the resources and activities that need to be modified for improving the workflow. The simulation model has increased the awareness of hospital employees regarding the complexity of the processes.

\section{REFERENCES}

1. Coiera E. Communication systems in healthcare. Clin Biochem Rev 2006;27(2):89-98.

2. Hendrich A, Chow MP, Skierczynski BA, et al. A 36-Hospital Time And Motion Study: How Do Medical-Surgical Nurses Spend Their Time? Permanente J 2008;12(3):25-34.

3. Pepino A, Torri A, Tamburis 0. Implementing simulationbased approaches for healthcare workflow analysis: the case of a department of laboratory medicine in South Italy. Proc. of the Third Intl. Conf. Advances in Computing, Communication and Information Technology- CCIT 2015; Institute of Research Engineers and Doctors, USA. ISBN: 978-1-63248-061-3 doi: 10.15224/ 978-1-63248-061-3-11 
De Rosa, Pepino, Giaconia, Guarino: Bedside Communication and Management of Vital Parameters and Alarms In CareIntensive Environments: Simulation Model Development for the Clinical Effectiveness Analysis of an Innovative Technology

4. Sendelbach S, Funk M. Alarm fatigue: A patient safety concern. AACN Adv Crit Care 2013;24(4):378-86.
5. Ventola CL. Mobile Devices and Apps for Health Care Professionals: Uses and Benefits. P T 2014;39(5):356-64.

6. Ascom Holding AG. Website. Available at: www.ascom. com. 